This manuscript is a preprint and has been submitted for publication in Journal of Computational Physics. Please note that, despite having undergone peer-review, the manuscript has yet to be formally accepted for publication. Subsequent versions of this manuscript may have slightly different content. If accepted, the final version of this manuscript will be available via the 'Peer-reviewed Publication DOI' link on the right-hand side of this webpage. Please feel free to contact any of the authors; we welcome feedback. 


\title{
Adaptive Mesh Refinement Strategies for a Novel Model of Immiscible Fluid Flow in Fractures
}

\author{
Sobhan Hatami ${ }^{\mathrm{a}}$, Stuart D.C. Walsh ${ }^{\mathrm{a}}$ \\ ${ }^{a}$ Department of Civil Engineering, Monash University, Melbourne, Australia
}

\begin{abstract}
In this paper, we consider two Adaptive Mesh Refinement (AMR) methods to simulate flow through fractures using a novel multiphase model. The approach represents the fluid using a two-dimensional parallel-plate model that employs techniques adapted from lattice-Boltzmann simulations to track the fluid interface.

Here, we discuss different mesh refinement strategies for the model and compare their performance to that of a uniform grid. Results from the simulations are demonstrated showing excellent agreement between the model and analytical solutions for both unrefined and refined meshes. We also present results from the study that illustrate the behaviour of the AMR front-tracking method. The AMR model is able to accurately track the interfacial properties in cases where uniform fine meshes would significantly increase the simulation cost. The ability of the model to dynamically refine the domain is demonstrated by presenting the results from an example with evolving interfaces.
\end{abstract}

Keywords: Multicomponent flow, Mesh refinement, Numerical Modelling

\section{Introduction}

2 Multiphase fracture flow is encountered in several different geo-engineering 3 contexts (geothermal energy [1, 2], oil and gas production [3, 4], carbon se4 questration [5, 6], and hydraulic fracturing [7, 8], for example). Nevertheless, 5 our understanding of flow through fractures remains limited - particularly when compared to our knowledge of multiphase flows in three-dimensional 7 pore networks. While experimental studies are invaluable in understanding 8 these systems, these studies are limited by the difficulties in monitoring the 
9

interface between the phases and detailed flow properties in controlled experimental conditions [9]. Thus, robust numerical models are required to gain insight into the behaviour of this class of fluid systems.

The ability of the lattice Boltzmann method (LBM) to simulate complex and moving boundaries, combined with its ease of implementation, has made it a popular method for modelling multi-phase flow [10, 11, 12, 13, 14]. There are several lattice Boltzmann methods to simulate such flow systems including interaction potential models [15], color-gradient models [16], freeenergy formulations [17], and kinetic-equation based models [18]. However, lattice Boltzmann modelling can be computationally expensive in fractured media, where the apertures and flow properties may vary by orders of magnitude [19]. This problem is compounded by the need to faithfully track the immiscible fluid interface - high-resolution lattice sizes are required to resolve the forces on the interface, adding to the computation cost.

In this paper, we use an intermediate model that explicitly represents the changes in the fracture aperture or flow properties, while implicitly solving the cross-sectional flow [19]. This model addresses many of the issues arising due to the difference in length scales between the fracture aperture and the in-plane flow. Nevertheless, the thickness of the immisicible fluid interface remains tied to the in-plane mesh resolution. Here we demonstrate how different Adaptive Mesh Refinement (AMR) strategies may be integrated into the model to alleviate the difficulty of resolving the fluid interface.

In AMR, refined meshes are introduced as needed in certain numericallysensitive parts of the simulation domain (e.g. the interface region), while coarser meshes are retained in other areas $[20,21]$. The mesh resolution is dynamically updated as the solution proceeds. This strategy retains numerical accuracy while reducing memory and time requirements [22, 20, 23].

Broadly speaking, there are three ways to implement the AMR: blockbased, cell-based, and patch-based [24, 25, 26]. In block-based (also known as tile-based) refinement, the domain is divided into several predefined nonoverlapping, fixed-size blocks [27, 28]. In this method, when a cell is tagged for refinement, the whole block which the cell belongs to is refined. In contrast, in cell-based refinement, the refinement strategy is implemented on a cell by cell basis which reduces the refined region [29]. Although the cellbased method avoids over refinement, it looses the advantage of using structured meshes. In patch-based AMR, cells that are tagged for refinement are grouped into a new (structured) level overlapping the coarse meshes [30, 31]. Thus, patch-based AMR retains much of the selectivity of cell based refine- 
ment, while benefiting from structured meshes similar to block-based techniques.

Here we compare the performance of cell-based and patch-based refinement and consider their implications for the novel immiscible-fluid fracture flow model [19]. The remainder of this paper is structured as follows: Section 2 is dedicated to the description of the immiscible mutiphase flow model and the implemented AMR techniques. In Section 3, the simulation results of multiphase flow with different AMR strategies are compared to each other and to those performed on uniform meshes. Where applicable, the results are compared to the available analytical solution to validate the accuracy of the model. In addition, the results of a case study is presented to show the capability and performance of the AMR model to dynamically refine the domain. The main findings and potential future works are summarized in the final section.

\section{Model Description}

The immiscible-fluid model used in this paper is based on the approach outlined in Walsh \& Carroll [19]. This model has three main components: I) a parallel plate model to provide the cross-sectional flow, II) a latticeBoltzman-like color-gradient model to provide phase separation, and III) a surface tension model to track the interface between the fluids. Below, we first provide a brief description of the modelling approach. We then discuss the AMR strategies used and how they were implemented into the model.

\subsection{Parallel plate model}

The general form of single phase flow through fracture can be expressed using the following equation [32]:

$$
\frac{\partial}{\partial x}\left(T_{x} \frac{\partial P}{\partial x}\right) d x+\frac{\partial}{\partial y}\left(T_{y} \frac{\partial P}{\partial y}\right) d y=\frac{\partial}{\partial t}(\rho V),
$$

in which $x$ and $y$ denote direction, $t$ is time, and $P, V, \rho$, and $T$ are pressure, volume, density, and transmissibility. Using the parallel plate model, transmisibility is calculated as [33]:

$$
T=\frac{h^{3} w}{12 \nu}
$$


where $h$ is fracture aperture, $w$ is fracture width, and $\nu$ is the kinematic viscosity. At the interface of two adjacent cells (e.g. $a$ and $b$ in Figure 1), the transmissibility is calculated using the harmonic average:

$$
T_{a b}=\frac{T_{a} T_{b}}{T_{a}+T_{b}}
$$
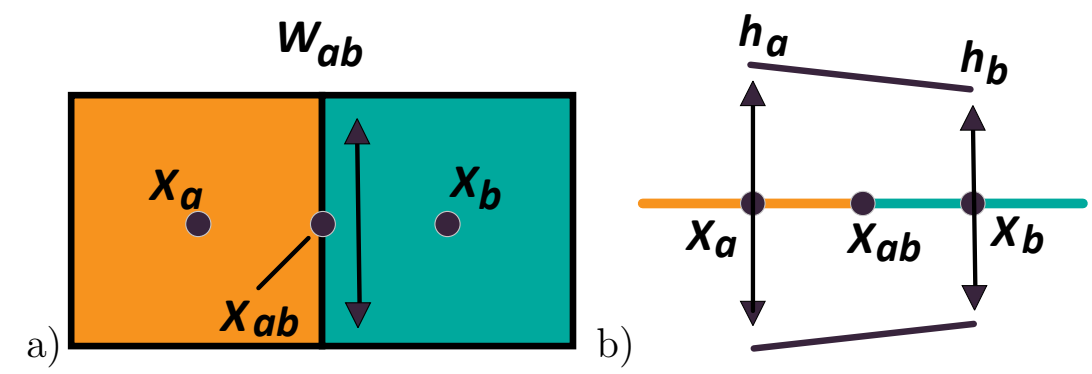

Figure 1: Top-down (a) and side (b) views of adjacent cells showing: the cell centers $x_{a}$ and $x_{b}$, the edge center $x_{a b}$, the edge length $w_{a b}$, and the cell apertures $h_{a}$ and $h_{b}$.

Multiphase flow in the fracture is simulated by coupling the parallel plate model with a recoloration technique. The separate fluid components are tracked using a color function, $\phi$, that represents the saturation of one of the fluid phases. The recoloration strategy allows the fluid components to moderately mix, producing a diffusive interface. In a lattice Boltzmann model, mixing of the components occurs naturally, as a consequence of the interactions between the packets used to represent fluid momentum components. Phase separation is then enforced through the recoloration scheme that segregates the two phases. However, there is no self-diffusion in parallel plate models. Instead the intrinsic mixing of lattice-Boltzmann fluid packets is replicated by introducing an artificial diffusive flux, $\delta \Phi^{d i f f}$, between adjacent cells. Once this is introduced, the same recoloration strategies used in lattice Boltzmann methods can be applied to the parallel plate flow model.

The recoloration strategy used here is based on that presented by LattvaKokko [34]. The strategy introduces a reflection term, $\beta$, that determines the interface width. For each timestep, the change in the color function consists of a diffusive component and an advective component:

$$
\delta \Phi_{a}=\sum_{b}\left(\delta \Phi_{a b}^{d i f f}+\delta \Phi_{a b}^{a d v}\right)
$$


For the adjacent cells $a$ and $b$ in Figure 1, the diffusive term is defined by

$$
\begin{aligned}
\delta \Phi_{a b}^{\text {diff }}=\frac{D}{w_{a b}^{2}}\left(\Phi_{b}\left[1-\beta\left(1-\Phi_{b}\right) \cos (\theta)\right]\right. & \\
& \left.-\Phi_{a}\left[1+\beta\left(1-\Phi_{a}\right) \cos (\theta)\right]\right) \delta t,
\end{aligned}
$$

where $D$ is the diffusion coefficient, $w_{a b}$ is the distance between the cells' center, $\beta$ is the anti-diffusion coefficient which controls the interface width, and $\theta$ is the angle between the color function gradient and the normal to the cell's face $\left(\right.$ i.e. $\left.\cos (\theta)=\frac{\nabla \phi \cdot \hat{n}}{|\nabla \phi|}\right)$.

An upwind scheme is used to calculate the advective term:

$$
\delta \Phi_{a b}^{a d v}=\frac{T_{a b}}{V}\left(P_{b}-P_{a}-\Delta P_{a b}\right) \phi_{a b}^{u p},
$$

where

$$
\phi_{a b}^{u p}=\left\{\begin{array}{ll}
\phi_{a} & \text { if } P_{b}-P_{a}-\Delta P_{a b} \leq 0 \\
\phi_{b} & \text { if } P_{b}-P_{a}-\Delta P_{a b}>0
\end{array} .\right.
$$

Here $V$ is the volume of the cell and $\Delta P_{a b}$ is the pressure differential at the interface. The pressure differential is determined by the surface tension, $\sigma$, the curvature $\kappa$, and the change in color function, $\Delta \phi$ :

$$
\Delta P_{a b}=-\sigma \kappa \Delta \phi
$$

The interface curvature, $\kappa$, is comprised of two parts, the out-of-plane curvature, $\kappa_{\perp}$, and the in-plane curvature, $\kappa_{\|}$:

$$
\kappa=\kappa_{\perp}+\kappa_{\|}=\frac{1}{r_{\perp}}+\frac{1}{r_{\|}},
$$

where $r_{\perp}$ and $r_{\|}$are the out-of-plane and in-plane radius of curvature, respectively. The out-of-plane radius of curvature is calculated as follows [35]:

$$
r_{\perp}=\frac{h}{2 \cos \left(\alpha_{w}+\alpha_{c}\right)},
$$

in which $h$ is the fracture aperture, $\alpha_{w}$ is the wetting angle, and $\alpha_{c}$ is the convergence angle of the fracture planes as illustrated in Figure 2. The inplane curvature (i.e. parallel to the fracture surface)

$$
\kappa_{\|}=-\nabla \cdot\left(\frac{\nabla \phi}{|\nabla \phi|}\right),
$$

is determined from the color function distribution using the MAC scheme provided in [36]. 


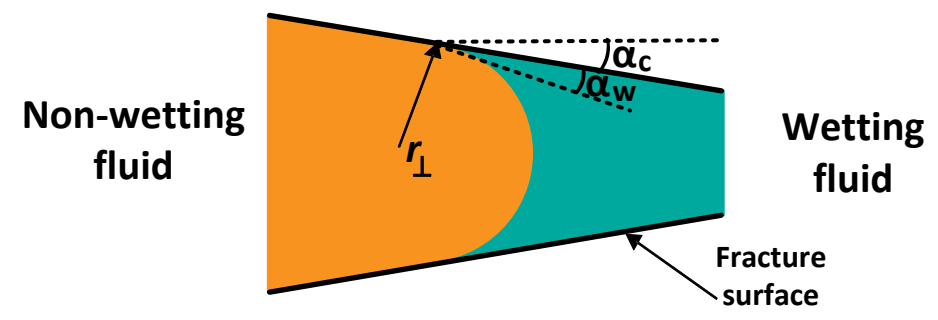

Figure 2: The radius of curvature normal to the fracture surface, $r_{\perp}$ is a function of wetting angle $\alpha_{w}$ and the angle of convergence $\alpha_{c}$.
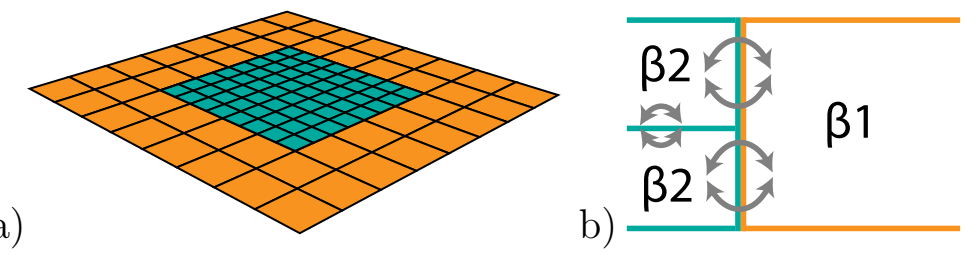

Figure 3: a) Mesh distribution in the cell-based AMR strategy where fine and coarse meshes are adjacent to each other. b) $\beta$ value is to be changed based on the length scale to provide smooth connection between meshes.

\subsection{AMR strategies}

Accurate simulation of highly unsteady-state fluid-flow problems, such as those involving shock waves or moving interfaces, requires a fine mesh resolution around key areas of interest. However, if a uniform mesh is used throughout, significant computational effort may be wasted on areas where nothing occurs. Adaptive mesh refinement provides a solution to this problem by refining the mesh in active regions while maintaining coarse meshes elsewhere. Here, we outline cell-based and patch-based refinement strategies for the immiscible multiphase flow model.

Figure $3 \mathrm{a}$ is an illustration of the mesh distribution in the cell-based refinement. In this case, the finer meshes lay adjacent to the coarser ones. Because individual cells are tagged for refinement, the footprint of the refined region is less than that for block-based refinement.

At coarse-fine mesh interfaces, the flux of particles should be adjusted to get a consistent flux across the two meshes. This can be accomplished by changing the value of $\beta$ - the anti-diffusion parameter controlling the number of particles crossing the interface (Figure $3 \mathrm{~b}$ ). Thus the key is to find a relationship between the length scale and $\beta$ to ensure consistency between the different mesh scales. 
a)

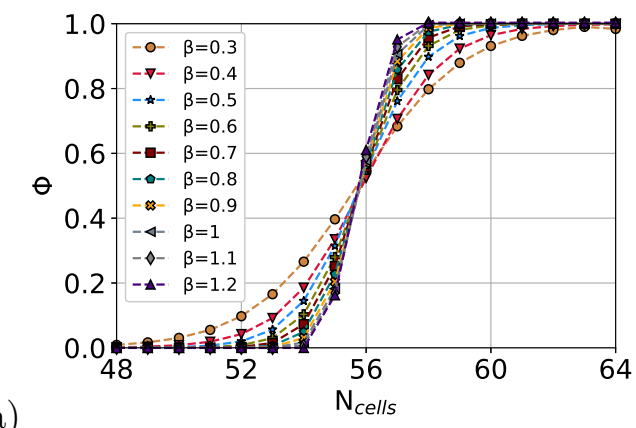

$\mathrm{N}_{\text {cells }}$

Figure 4a shows the shape of the fully developed interface for different $\beta$ values ranging from 0.3 to 1.2 using a uniform mesh structure throughout the domain. Like the other diffusive-interface methods [37, 38], use of a color function causes the interface to spread over a portion of the domain. Larger $\beta$ values result in a greater portion of the color particles being reflected over the cell length - producing a narrower interface.
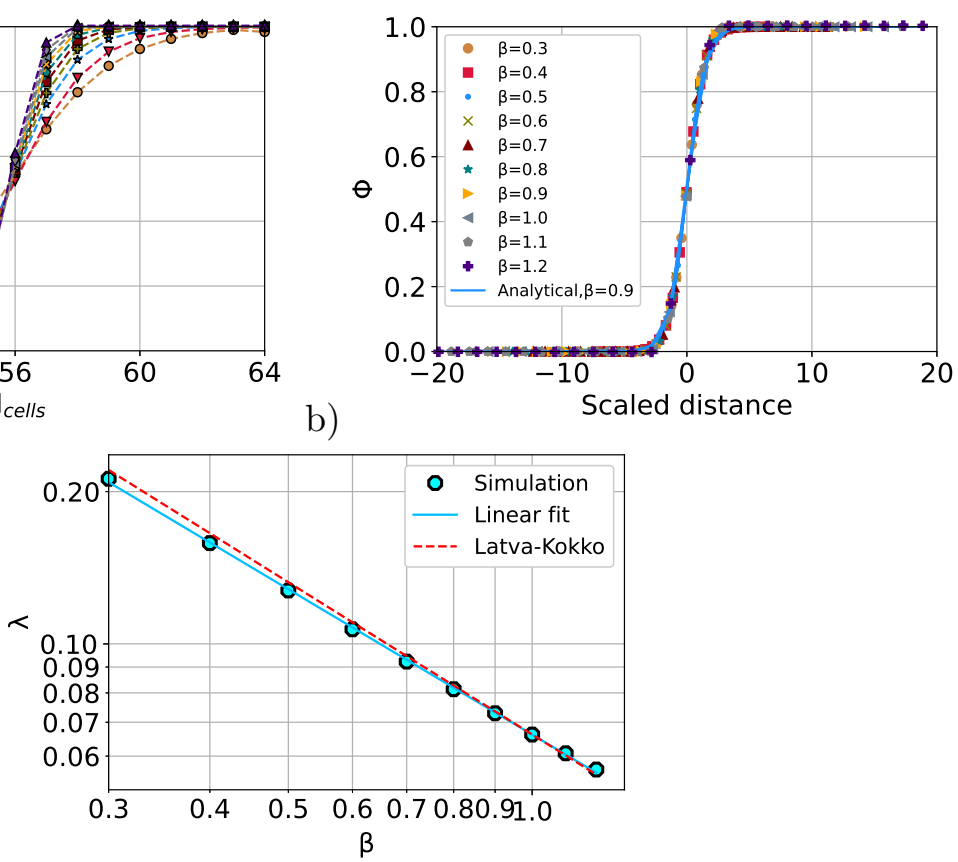

c)

Figure 4: Effect of $\beta$ on interface width: a) Fully developed interface profiles for different $\beta$ values versus cell's number, b) The same profiles as in figure a and the profile for quasi-analytical solution with $\beta=0.9 \mathrm{c}$ ) Length scale, $\lambda$, versus $\beta$ profile along with the Latva-Kokko quasi-analytical solution.

Latva-Kokko [34] derived a quasi-analytical solution which describes the shape of the fully developed interface:

$$
\begin{aligned}
\phi & =\frac{e^{k(\beta)\left(x-x_{0}\right)}}{1+e^{k(\beta)\left(x-x_{0}\right)}} \\
k(\beta) & =\frac{2}{3}(1+\sqrt{2}) \beta,
\end{aligned}
$$

in which $\phi$ is colour function, $x-x_{0}$ is the distance from the interface location, and $\beta$ is the anti-diffusion coefficient. The interface profiles in Figure 4a were 
fitted to the quasi-analytical interface profile corresponds to $\beta=0.9$ (see Figure 4b), yielding the following empirical equation:

$$
\ln (\lambda)=-0.957677 \ln (\beta)-2.578139,
$$

where $\lambda$ denotes the length scale. Figure 4c illustrates the length scale, $\lambda$, and $\beta$ relationship from the simulation results along with those obtained from the linear fit and quasi analytical solution. As can be seen, the quasi-analytical solution deviates from the simulation results. Although this inconsistency is small, capturing the non-linearity is important to accurately match the interface behaviour at different scales. Hence, equation 14 was incorporated into the model to change the $\beta$ values according to the length scale in the case of cell-based refinement.

The patch-based refinement was conducted using the AMReX library [39]. AMReX provides a framework for building parallel, block-structured AMR for solving system of partial differential equations (PDEs). Figure 5a illustrates an example of mesh distribution for patch-based strategy where a finer level is created on top of the coarser level out of the patches tagged for refinement. Each patch contains the ghost cells storing the necessary information from the neighbours needed for computation. A hierarchical sub-cycling with time algorithm is used - in which the finer level is advanced with smaller time step than the coarse level. For example, as is shown in Figure 5b, if the new level 2 is refined by a factor of two, it advances for two steps with each time step half of that for the coarser level. Finally the two levels are synchronized and the color distribution is updated throughout the domain. The sub-cycling process removes the need for a $\lambda-\beta$ relationship like the earlier model.

\section{Simulation results}

In this section, simulation results for the cell-based and patch-based refinement strategies are presented and compared to those from uniform meshes. Next an example is given showing how the model can accurately track the interfacial properties of small droplets where using the uniform meshes deviates significantly from the analytical solution. Finally, a case study is presented showing the ability of the model to dynamically refine the domain as the interface moves across the fracture space.

Figure 6a shows the steady state color distribution profile of a droplet simulated on a uniform mesh. The radial distribution of the colour function 

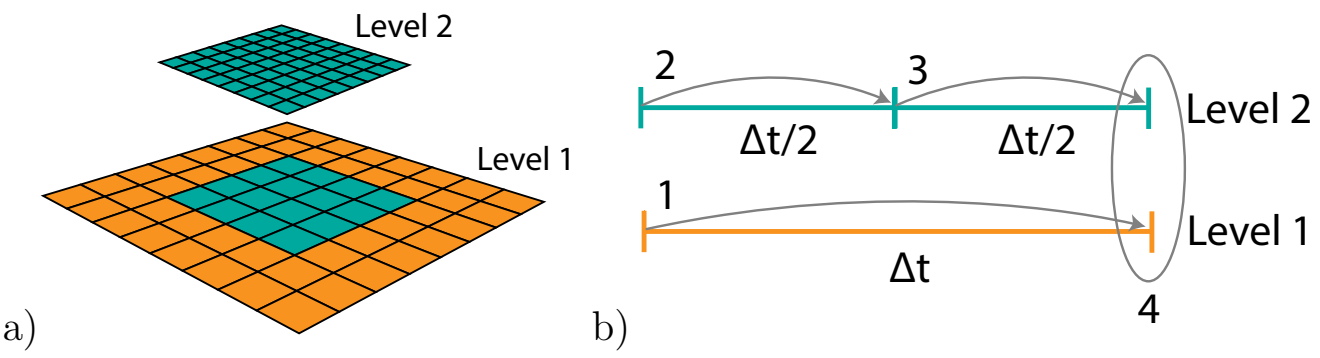

Figure 5: Mesh distribution in overlapping mesh refinement strategy. A finer level is defined on the top of the coarser level. b) The sub-cycling stages when the new level is refined by a factor of two: 1) integrate level 1 over $\Delta t, 2,3)$ integrate level 2 over $\Delta t / 2,4$ ) synchronize levels 1 and 2 .

a)

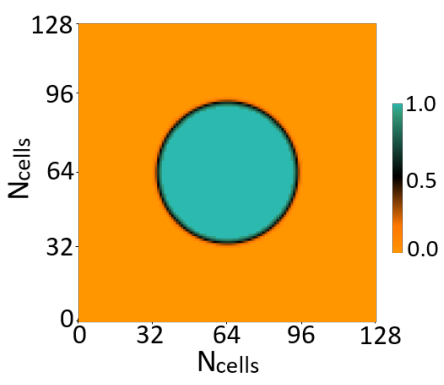

Figure 6: a: Top view of the colour function distribution at the steady state condition for $\beta=0.9$. b: Radial distribution of the color function versus the cell's number.

over a horizontal cross section passing through the center of the droplet is illustrated in Figure 6b. As already mentioned, the width of the interface is governed by $\beta$ parameter. For this paper the typical $\beta$ value of 0.9 [34] was used unless explicitly mentioned otherwise. Periodic boundary conditions applied to both $x$ and $y$ boundaries. Two separate sets of simulations were conducted to test both the cell-based refinement and the patch-based refinement strategies.

The first set of simulations evaluated the cell-based refinement strategy. Three different cases were considered to test if the same interface profiles can be generated using $\lambda-\beta$ relationship (equation 14):

1. In the first case, the cell size is uniform throughout the domain;

2. In the second, the cell size in half of the interface region was refined by a factor of two; and 
a)

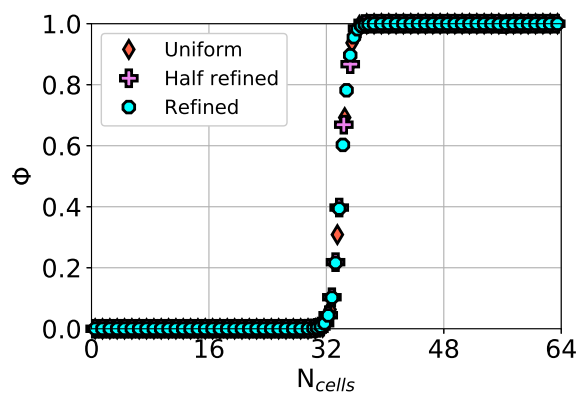
relationship.

3. In the third, all of the interface was refined by a factor of two.

For the first case, $\beta=0.9$ was used in all cells, while in the last two cases, $\beta$ was changed in the refined cells according to Equation 14. The three simulations began with the same initial conditions and were advanced until the steady state condition was achieved. The results of these simulations are presented in Figure 7a. The three plots are in good agreement, demonstrating the smooth communication between the different levels provided by the $\lambda-\beta$

Figure 7: Steady-state interface profile versus cell's number for uniform and refined meshes: a) cell-base AMR; b) patch-based AMR.

Two cases are considered in the second example, which demonstrates the patch-based refinement strategy. In the first, uniform meshes were used throughout the domain and in the second, a refined region was defined on top of the coarse level just at the droplet region. As is presented in Figure 7b, the fully developed interface profiles for both cases match quite well which confirms the accuracy of the patch-based refinement strategy.

As noted earlier, one of the main challenges in immiscible multiphase flow is tracking the interface properties between the phases. Fine mesh resolution is normally needed to accurately resolve the forces exerting on the interface which can make the simulation computationally expensive, particularly when only a small portion of the domain is occupied by one of the phases. In these cases using AMR can significantly cut the simulation costs by selectively refining the domain as is depicted in Figure 8.

Figure 9a shows the pressure differential across the boundary of a single droplet defined on a uniform mesh as a function of the droplet radius. Here $r_{D}$ is dimensionless droplet radius which is defined as the droplet radius divided by the length of the coarsest mesh $\left(r_{D}=\frac{r}{l}\right.$ where $r$ is droplet radius and 


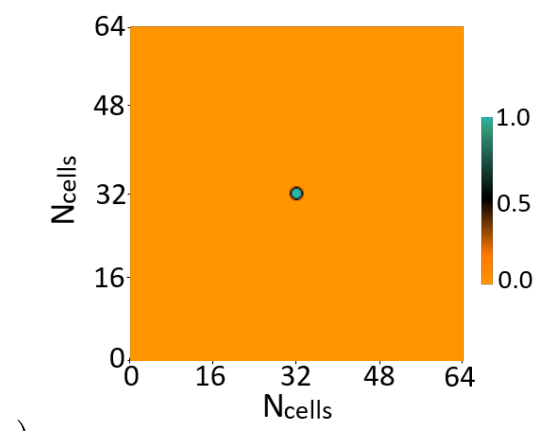

a)

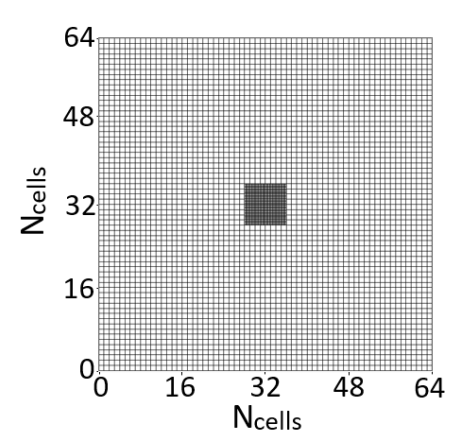

b)

Figure 8: a) Colour distribution profile for a small droplet with the radius equal to the length of a coarse cell. b) A refined level is created on top of the coarse level at the droplet region with cell sizes that are four times smaller.

$l$ is length of the coarsest cell). The value of $r_{D}=1$ means that the droplet radius is equal to the size of the coarsest mesh. The simulation results are compared to the Young-Laplace analytical solution for the capillary pressure across the interface of two static phases:

$$
\Delta P=-\sigma\left(\frac{1}{r_{1}}+\frac{1}{r_{2}}\right),
$$

where $\sigma$ is the interfacial tension and $r_{1}$ and $r_{2}$ are the principal radii of curvature. As can be seen in the figure, for the droplet radius smaller than four coarse grid size, the simulation results deviate from the analytical solution which is similar to what is observed in similar lattice Boltzmann models with diffusive interfaces $[34,37,22,19]$. When the droplet radius equals the size of the coarse simulation cell, the the analytical solution and the simulation results differ by more than $120 \%$. Figure $9 \mathrm{~b}$ shows the AMR simulation results for similar droplet radii as in Figure 9a. A refined level is defined on top of the coarse level at the droplet boundary (see Figure 8). This provides sufficient resolution to accurately capture the interface properties. In this case, even for the smallest droplet radius, the simulation results are in good agreement with the analytical solution.

In the final example, merging droplets are considered to test the ability of the model in dynamic tracking of moving interfaces. Using the patch-based AMR model, four droplets with different radius were introduced at the center of the domain (see Figure 10a). Figure 10b shows the mesh distribution over the domain. In this case two refined levels were considered. The first 

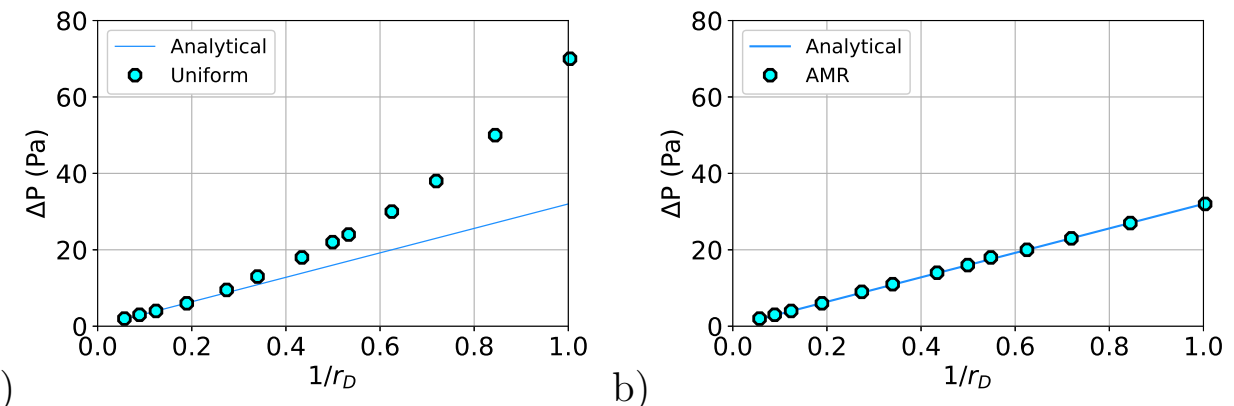

Figure 9: Pressure differential $(\Delta P)$ as a function of droplet radius for neutrally wetting fracture surface $\left(\alpha_{w}=90^{\circ}\right)$, fracture aperture of $1 \mu \mathrm{m}$, and surface tension of $50 \mathrm{dyne} / \mathrm{cm}$ : a) uniform mesh, b) AMR. The solid line shows Young-Laplace analytical solution.

level was set to be defined at the regions with $0.01<\phi<0.99$ and the second one at the regions with $0.2<\phi<0.8$. The color distribution profile along a horizontal line through the center of the domain is also depicted in Figure 10c. As Figure 10d illustrates the interfaces evolve as the simulation proceeds and the smaller droplets merge into the largest one. Figure 10e and Figure 10f show the updated refined levels and the color profile, respectively. The simulation proceeded until the steady state condition was achieved. At this point, a single droplet was created out of the small droplets as is depicted in Figure 10g along with the corresponding mesh distribution in Figure 10h and color profile in Figure 10i. This demonstrates the ability of the AMR model to dynamically track evolving interfaces. As the figures show, the refined levels are continuously updated in accordance to the new color distribution to accurately capture the interfacial properties - providing sufficient resolution in those regions and reducing the total computational cost.

\section{Conclusions}

Accurate tracking of interfacial properties remains a key challenge when simulating immiscible multiphase flows in fractures. A fine uniform-mesh defined throughout the domain can accurately resolve the forces exerting on fluid interfaces. However, this strategy is computationally inefficient, as a coarser mesh resolution is often sufficient for most of the domain.

Adaptive Mesh Refinement offers a solution to this problem by dynamically increasing the mesh resolution as needed in different parts of the domain. Thus the simulation accuracy is retained for reduced computational 
a)

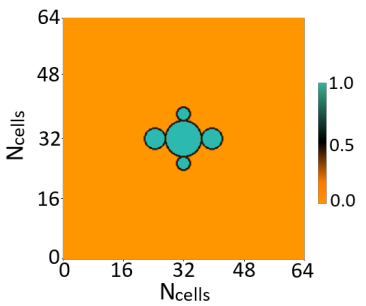

d)

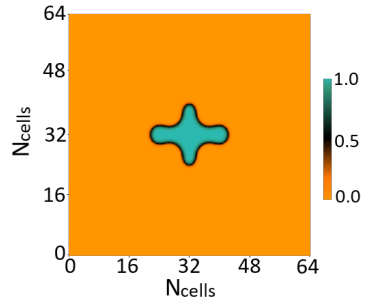

e)

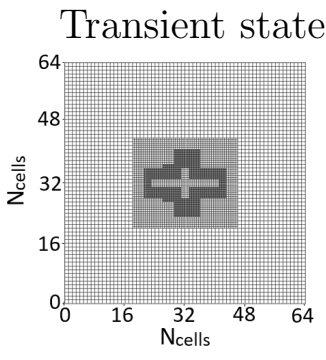

Steady-state distribution

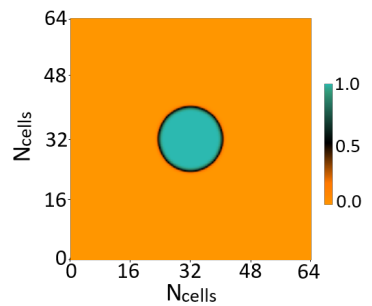

g)

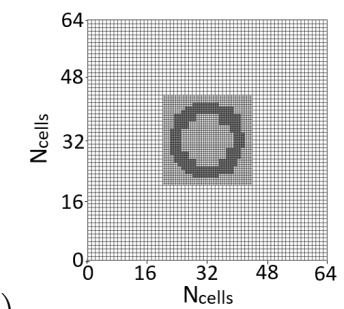

h)

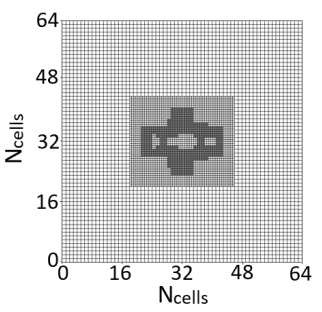

c)

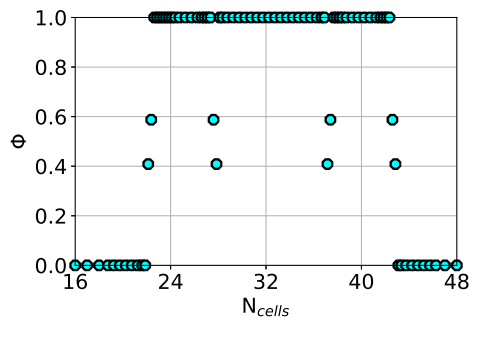

f)

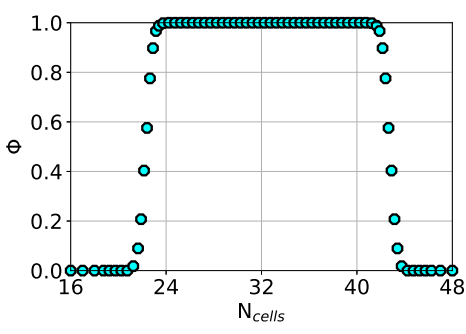

Figure 10: Different stages in a simulation tracking merging droplets (wetting angle $\alpha_{w}=$ $60^{\circ}$, fracture aperture $h=1 \mu \mathrm{m}$, and surface tension $\sigma=50$ dyne $/ \mathrm{cm}$ ). a) shows the initial $2 \mathrm{D}$ color distribution profile, b) shows the corresponding mesh distribution, and c) shows the color distribution along the center line. Figures d), e) and f) show the same plots shortly after the start; while Figures g), h), and i) show the steady state distributions. 
cost. In this paper, cell-based and patch-based AMR strategies were incorporated into a model that describes immiscible fracture flows by combining a parallel-plate model with an lattice Boltzmann recoloration scheme.

The cell-based refinement strategy was implemented by introducing a mathematical expression to relate the length scale to the anti-diffusion parameter that controls the interface width. In the patch-based refinement, a recursive hierarchical sub-cycling in time is used where the finer level advances with smaller time step than the coarser level. The accuracy of both methods was successfully tested by considering different cases and comparing the results to those from uniform meshes.

For small droplets where uniform meshes are unable to resolve the interface properties, the AMR model is able to successfully produce the same results as those of the analytical solution. Finally, the ability of the model to dynamically refine the domain was illustrated through an example in which small droplets are joined together to create a large droplet. In this case, as the interfaces evolved with time, the refined levels were updated based on the new color distribution to both provide sufficient resolution at the interface and reduce the computational effort.

\section{References}

[1] G. Feng, X. Wang, M. Wang, Y. Kang, Experimental investigation of thermal cycling effect on fracture characteristics of granite in a geothermal-energy reservoir, Engineering Fracture Mechanics 235 (2020) 107180.

[2] S. Molins, D. Trebotich, B. Arora, C. I. Steefel, H. Deng, Multi-scale model of reactive transport in fractured media: diffusion limitations on rates, Transport in Porous Media 128 (2019) 701-721.

[3] M. HosseiniMehr, M. Cusini, C. Vuik, H. Hajibeygi, Algebraic dynamic multilevel method for embedded discrete fracture model (f-adm), Journal of Computational Physics 373 (2018) 324-345.

[4] I. Y. Akkutlu, Y. Efendiev, M. Vasilyeva, Y. Wang, Multiscale model reduction for shale gas transport in poroelastic fractured media, Journal of Computational Physics 353 (2018) 356-376.

[5] J. Iyer, S. D. Walsh, Y. Hao, S. A. Carroll, Assessment of two-phase flow on the chemical alteration and sealing of leakage pathways in cemented 
wellbores, International Journal of Greenhouse Gas Control 69 (2018) $72-80$.

[6] F. Ren, G. Ma, Y. Wang, L. Fan, H. Zhu, Two-phase flow pipe network method for simulation of co2 sequestration in fractured saline aquifers, International Journal of Rock Mechanics and Mining Sciences 98 (2017) $39-53$.

[7] J. Mohan, G. Pope, M. Sharma, Effect of non-darcy flow on well productivity of a hydraulically fractured gas-condensate well, SPE Reservoir Evaluation \& Engineering 12 (2009) 576-585.

[8] J. Napier, E. Detournay, An unstructured mesh algorithm for simulation of hydraulic fracture, Journal of Computational Physics 419 (2020) 109691.

[9] R. L. Detwiler, H. Rajaram, R. J. Glass, Interphase mass transfer in variable aperture fractures: Controlling parameters and proposed constitutive relationships, Water resources research 45 (2009).

[10] M. C. Sukop, D. Or, Lattice boltzmann method for modeling liquidvapor interface configurations in porous media, Water Resources Research 40 (2004).

[11] M. E. McCracken, J. Abraham, Multiple-relaxation-time latticeboltzmann model for multiphase flow, Physical Review E 71 (2005) 036701.

[12] J. Bao, L. Schaefer, Lattice boltzmann equation model for multicomponent multi-phase flow with high density ratios, Applied Mathematical Modelling 37 (2013) 1860-1871.

[13] H. Huang, M. Sukop, X. Lu, Multiphase lattice boltzmann methods: Theory and application (2015).

[14] Y. Wang, T. Chung, R. T. Armstrong, J. McClure, T. Ramstad, P. Mostaghimi, Accelerated computation of relative permeability by coupled morphological and direct multiphase flow simulation, Journal of Computational Physics 401 (2020) 108966. 
[15] X. Shan, H. Chen, Lattice boltzmann model for simulating flows with multiple phases and components, Physical review E 47 (1993) 1815.

[16] A. K. Gunstensen, D. H. Rothman, Microscopic modeling of immiscible fluids in three dimensions by a lattice boltzmann method, EPL (Europhysics Letters) 18 (1992) 157.

[17] M. R. Swift, E. Orlandini, W. Osborn, J. Yeomans, Lattice boltzmann simulations of liquid-gas and binary fluid systems, Physical Review E 54 (1996) 5041.

[18] Z. Guo, T. Zhao, Finite-difference-based lattice boltzmann model for dense binary mixtures, Physical Review E 71 (2005) 026701.

[19] S. D. Walsh, S. A. Carroll, Fracture-scale model of immiscible fluid flow, Physical Review E 87 (2013) 013012.

[20] K. Schmidmayer, F. Petitpas, E. Daniel, Adaptive mesh refinement algorithm based on dual trees for cells and faces for multiphase compressible flows, Journal of Computational Physics 388 (2019) 252-278.

[21] B. Runnels, V. Agrawal, W. Zhang, A. Almgren, Massively parallel finite difference elasticity using block-structured adaptive mesh refinement with a geometric multigrid solver, Journal of Computational Physics 427 (2021) 110065.

[22] Z. Yu, L.-S. Fan, An interaction potential based lattice boltzmann method with adaptive mesh refinement (amr) for two-phase flow simulation, Journal of Computational Physics 228 (2009) 6456-6478.

[23] O. Antepara, N. Balcázar, A. Oliva, Tetrahedral adaptive mesh refinement for two-phase flows using conservative level-set method, International Journal for Numerical Methods in Fluids (2020).

[24] B. T. Gunney, R. W. Anderson, Advances in patch-based adaptive mesh refinement scalability, Journal of Parallel and Distributed Computing 89 (2016) 65-84.

[25] C. Liu, C. Hu, Block-based adaptive mesh refinement for fluid-structure interactions in incompressible flows, Computer Physics Communications 232 (2018) 104-123. 
[26] A. Giuliani, L. Krivodonova, Adaptive mesh refinement on graphics processing units for applications in gas dynamics, Journal of Computational Physics 381 (2019) 67-90.

[27] L. Ivan, Development of high-order CENO finite-volume schemes with block-based adaptive mesh refinement, 2011.

[28] F. Golay, M. Ersoy, L. Yushchenko, D. Sous, Block-based adaptive mesh refinement scheme using numerical density of entropy production for three-dimensional two-fluid flows, International Journal of Computational Fluid Dynamics 29 (2015) 67-81.

[29] H. Ji, F.-S. Lien, F. Zhang, A gpu-accelerated adaptive mesh refinement for immersed boundary methods, Computers \& Fluids 118 (2015) 131147.

[30] C. Pantano, R. Deiterding, D. J. Hill, D. I. Pullin, A low numerical dissipation patch-based adaptive mesh refinement method for large-eddy simulation of compressible flows, Journal of Computational Physics 221 (2007) 63-87.

[31] C. R. Ferreira, M. Bader, Load balancing and patch-based parallel adaptive mesh refinement for tsunami simulation on heterogeneous platforms using xeon phi coprocessors, in: Proceedings of the Platform for Advanced Scientific Computing Conference, pp. 1-12.

[32] S. Sarkar, M. N. Toksoz, D. R. Burns, Fluid flow modeling in fractures, Technical Report, Massachusetts Institute of Technology. Earth Resources Laboratory, 2004.

[33] C. Neuzil, J. V. Tracy, Flow through fractures, Water Resources Research 17 (1981) 191-199.

[34] M. Latva-Kokko, D. H. Rothman, Diffusion properties of gradient-based lattice boltzmann models of immiscible fluids, Physical Review E 71 (2005) 056702.

[35] R. J. Glass, H. Rajaram, R. L. Detwiler, Immiscible displacements in rough-walled fractures: Competition between roughening by random aperture variations and smoothing by in-plane curvature, Physical Review E 68 (2003) 061110. 
[36] J. U. Brackbill, D. B. Kothe, C. Zemach, A continuum method for modeling surface tension, Journal of computational physics 100 (1992) $335-354$.

[37] L. Wu, M. Tsutahara, L. S. Kim, M. Ha, Three-dimensional lattice boltzmann simulations of droplet formation in a cross-junction microchannel, International journal of multiphase flow 34 (2008) 852-864.

[38] I. Halliday, A. Hollis, C. Care, Lattice boltzmann algorithm for continuum multicomponent flow, Physical Review E 76 (2007) 026708.

[39] W. Zhang, A. Almgren, V. Beckner, J. Bell, J. Blaschke, C. Chan, M. Day, B. Friesen, K. Gott, D. Graves, et al., AMReX: a framework for block-structured adaptive mesh refinement, Journal of Open Source Software 4 (2019). 